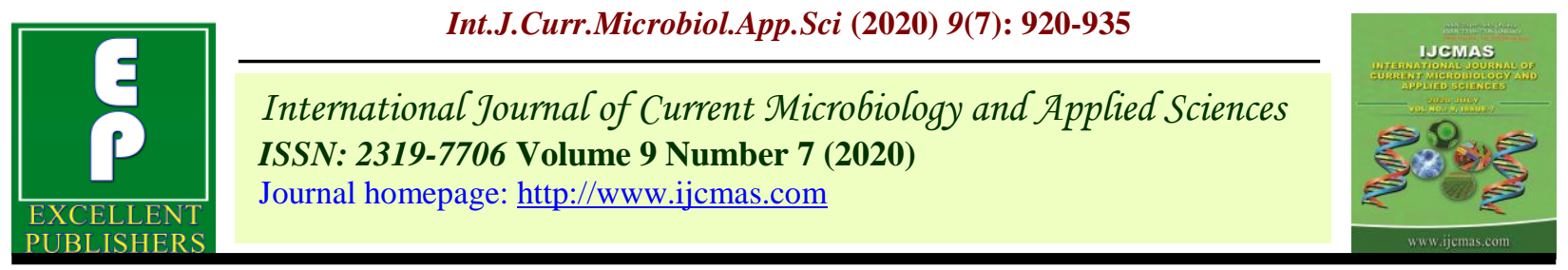

\title{
Effect of Short Term Waterlogging on Plant Morphology, Chlorophyll and Carotenoid Content of Tomato (Solanum lycopersicum L. Mill) during Vegetative Stage
}

\author{
A. Mohanty, R. K. Panda*, G. R. Rout, K. C. Muduli and P. Tripathy \\ Department of Plant Physiology, College of Agriculture, OUAT, Bhubaneswar-751003, \\ Odisha, India \\ *Corresponding author
}

\begin{tabular}{l} 
Ke y w o r d s \\
Waterlogging, \\
Tomato, \\
Chlorophyll, \\
Carotenoid, Leaf \\
area, Total biomass \\
\hline Article Info \\
\hline $\begin{array}{l}\text { Accepted: } \\
11 \text { June } 2020 \\
\text { Available Online: } \\
10 \text { July } 2020\end{array}$ \\
\hline
\end{tabular}

An experiment was arranged in a factorial completely randomized design with an aim to investigate the effect of short term waterlogging on four popular Khariftomato cultivars of Odisha (Utkal Pallavi, Utkal Dipti, Utkal Kumari and Utkal Pragyan). 45 days old tomato plants were subjected to $15 \mathrm{~cm}$ of stagnant water for a period of 0,1,2 and 3 days. High survivability under 1 and 2 days of waterlogging were recorded in U.Kumari of about $80 \%$ and $60 \%$ respectively because of high adventitious root formation, whereas all the plants died under 3 days of waterlogging due to root hypoxia. Under short term waterlogging for 2days U.Pragyan was highly affected showing a maximum decrease in plant height, leaf area and other morphological character with respect to control and other cultivars. Leaf Chlorophyll and carotenoid content were evaluated in each seven days interval after de- waterlogging. There was recorded a significant decrease in total chlorophyll and carotenoid content under water logging. A least about $8.9 \%$ and $5.7 \%$ decrease in chlorophyll and carotenoid respectively was observed in U.Kumari after 1 day of waterlogging. The shoot, root dry weight as well as total biomass of the plants were tremendously decreased under waterlogging.

\section{Introduction}

Waterlogging is one of the major abiotic stress and major constraint on crop growth and productivity ultimately results into plant death (Jackson and Colmer 2005, Voesenek et al., 2006). Adverse impact of climate change is likely to increase the incidence of flooding and waterlogging (Bailey-Serres and Voesenek, 2008). Soil is considered as waterlogged if the standing water layer on the soil surface is at least $20 \%$ higher than the field capacity of soil (Aggarwal et al., 2006). It is estimated that about $13 \%$ of the global land area and $16 \%$ of the tomato areas in production worldwide are prone to the risk of flooding and water logging (Ahsana et al., 2007).

Tomato is a moderate nutritional crop and is considered as important source of and vitamin $\mathrm{c}$ and minerals. The tomato fruit contains significant amount of lycopene, beta carotene, Magnesium, Potassium, niacin and riboflavin. 
It has antioxidant properties and potential beneficial health effects (Zheng et al., 2010). About 150 million tons of tomatoes were produced in the world and India is the 2nd largest producer of tomatoes, producing nearly 17.5 million tons and the area under cultivation was 5.4 million ha with average production of 15.68 qha-1 (Department of Agriculture and Co-operation 2016-17).

In many Asian countries, especially in India the typhoon-caused heavy rains often cause tomato production (Lin et al., 2004).Some species of crops, such as rice, are particularly tolerant to submergence, whereas others, such as tomato (Solanum lycopersicon L.), are highly intolerant (Ahsan et al., 2007). Tomato is highly sensitive to waterlogging, with in a period of 48 hours the plants start showing epinasty curvature in middle leaves.

As a consequence of disturbed physiological functioning, vegetative and reproductive growth of tomato plant is negatively affected by water logging (Kozlowski, 1997; Gibbs and Greenway, 2003). Water logging cause yellowing and death of the leaves from the lower ones to the stem, epinasty in tomato leaves (Kramer, 1951), decrease in the nitrogen concentration in shoots of plant. Seedlings can occur rapidly after the onset of water logging and precede leaf chlorosis (Drew and Sisworo, 1977; Wang et al., 1996) and consequently reduces shoot and root growth, dry matter accumulation, and final yield (Kozlowski, 1997; Drew, 1992, Huang et al., 1994a, 1994b; Malik et al., 2002).

Under water logging gas diffusion rate in water and oxygen availability to plant decreases up to $10-{ }^{4}$ fold. The adverse effects of water logging shows degradation in chlorophyll content, yellowing of leaves and detection of electrolytic leakage in tomato leaves (Yan et al., (2006). Water logging also causes adventitious root development
(Jackson, 2002, Voesenek et al., 2006, Bailey-Serres and Voesenek, 2008). Tolerant genotypes to waterlogging are associated with a complex trait linked to many morphological and physiological adaptations under adverse condition.

In order to increase the productivity under adverse circumstances tolerant genotypes are required with desired traits like hypertrophied lenticels, aerenchyma tissue, and adventitious roots (Kozlowski, 1997).

Keeping that in mind the objective of the study was to understand the morphological and some physiological behaviour of four tomato genotypes in waterlogging to may lead to identify tolerating genotype in vegetative stage.

\section{Materials and Methods}

4 sets of pots were used to grow tomato seedlings having 4 popular kharif varieties of Odisha (1.Utkal Pallavi, 2. Utkal Dipti 3. Utkal Kumari 4. Utkal Pragyan). The potsof $25 \mathrm{~cm}$ depth, $20 \mathrm{~cm}$ diameter were used with one seedling per pot. The dose of fertilizer (120:60:50 NPK kg/ha) was applied taking soil weight into consideration (1 ha of land soil $=2.24 \times 106 \mathrm{~kg}$ of soil) of each pot.Out of 4 sets, one set with all the four tomato varieties kept as such under normal condition and other three were imposed to stress under $15 \mathrm{~cm}$ of water level at 45 DAT for 0 days (S0), 1 day (S1), 2days (S2) and 3 days (S3).

After removal of water logging condition the recovery $\%$ was taken and other observations were taken after 2 days of water logging in each 7days interval up to 3 successive weeks. Individual plants were scored by using a modified scale of 0-5 by Yeboha et al., 2008 for effect of wilting, where 0 is a dead plant, 1 is for $100-75 \%$ of wilt from tip to the base, 2 
scores for $74-50 \%$ wilting of leaves from tip to the middle, 3 for leaves between base and middle undulating, 4is for recurved leaves margins and 5 is for green plant with no sign of stress.

0-3 scale score for adventitious root formation (ARF) under waterlogging was done visually (Yeboha et al., 2008 ) 0 = no ARF, 1= low, $2=$ medium, $3=$ high and yellow leaf percentage was determined by using by using a scale of 1-6 score (modified from Mohanty and Ong 2003) where 1 scores for no yellow leaves, 2 is for $10-30 \%$ of leaves were yellow, 3 is for $30-50 \%$ of leaves were yellow, 4 is for $50-70 \%$ were yellow, 5 is for when most leaves were yellow, 6 is for when all leaves were yellow.

Plant height $(\mathrm{cm})$, number of leaves per plant and leaf area $\left(\mathrm{cm}^{2}\right)$ of 2nd top leaf was recorded after withdrawal of stress .The dry weight of the shoot and root were recorded separately from each treatment after harvesting and expressed in gram dry weight per plant.

The total biomass of the plant parts such as stem, leaves, branches, roots along with the weight of the fruits were recorded from each treatment after harvesting and expressed as gram wt. per plant. Estimation of chlorophyll by non-destructive SPAD (Hansatech, Model CL-01) and destructive (Arnon 1949) method was done.

In destructive method the third leaf was taken and immediately kept in moist polythene bags to keep them fresh. 100 grams of fresh leaf was taken from the middle portion of the leaf and were cut into small pieces. The leaf discs were then put in $80 \% \mathrm{v} / \mathrm{v}$ acetone solution and kept in dark for 24 hours. The absorbance (OD) at $645 \mathrm{~nm}$ and $663 \mathrm{~nm}$ was recorded for both chlorophyll and carotenoid content of leaves.

\section{Results and Discussion}

\section{Survival percentage}

Survivability is a critical criterion to differentiate between tolerant and susceptible. Two days after removal of waterlogging the survival percentage of the plants were recorded after recovery. All the plants of all cultivars under 3 days of waterlogging stress were died whereas, under 2 days of waterlogging highest survival percentage of about $60 \%$ and $80 \%$ were recorded in U.Kumari under 2days and 1 day of waterlogging, whereas in U.Pragyan it was less than $33 \%$ and $42 \%$ under 2 and 1day of stress represented in figure number 1 .

\section{Leaf chlorosis, adventitious root formation (ARF) and wilting percent}

The individual plants was scored for tolerance after de-water logging (15 cm stagnant water for 1 day and 2 days) using a modified scale of 0-5 for wilting, 0-3 for adventitious root formation and 1-6 in terms of yellow leaf percentage and represented in Figure number 2,3 and 4 respectively.

Walter et al., 2004 and Mano and Omori 2005 reported that Tomato had adventitious root growth under water logging condition and helps the root system to obtain oxygen directly from the air and allows the plant to absorb nutrients from the soil, which supported the data recorded on 2 days after recovery from water logging and on above score revealed that U.Kumari had maximum number of adventitious roots followed by U.Dipti and U.Pallavi than control with a range of high to medium. This characteristics plays an important role in adaptation to flooding condition. U. Kumari had waterlogged tolerance ability in a way of producing more adventitious roots that might compensate for seminal root dysfunction in 
hypoxia condition for better water and mineral uptake The cultivar showing highest percent of yellow leaves was observed in both U.Pragyan and U.Pallavi followed by U.Dipti but U.Kumari had less percent of yellow leaf than the above cultivars. The same finding was supported by Vincent et al., 2010. Dawood T. 2005 et al., and Kramer 1951 that the yellowing and death of the lower leaves of the tomato plants is associated with by desiccation because of anaerobic conditions and production of toxic compounds.

This was hypothesized that the dried and dead cells of roots might have produce toxic and reduced substances like nitrates and sulphates and might have infected with microbes. Thus it can be concluded that with lower percentage of yellow leaves U.Kumari showed better adaptation to waterlogging. Moreover, in U.Kumari curled leaves appeared in between 2 days of waterlogging, whereas there was 50-100\% damage recorded in wilting percentage scoring. The present finding was also in line of the previous work and more significantly the cultivar.

\section{Plant height}

Plant height was measured after 2 days, 9 days, 16 days and 23 days of de-water logging revealed that all the tomato cultivars were having less plant height than control condition and depicted in Table number 1. Maximum decrease in the plant height was recorded in cultivar U.Pragyan up to 9 days after de-water logging followed by U.Kumari up to 16 days. Significant decrease in plant height was recorded in all the treatments among the varieties and water stress as well as their interaction. The maximum decrease in plant height was with a tune of $23.1 \%$ and $22 \%$ in U.Dipti under 2 days and 1 day of water logging respectively. A minimum percent decrease in height was observed in U. Kumari of $15.3 \%$ and $18.9 \%$ under 1 day and 2 days of water logging over control. Though the variation in plant height is depending up on their genetic potentiality to the environmental factors but reduction in plant height under stagnant water conditions is a stress factor and the results also corroborates with the work of Walter, 2004 and Vincent E. et al., 2010. Reduced plant growth due to flooding was also observed in Annona species (NunexElisea, 1999), Panicum antidotale (Ashraf, 2003), Paspalum dilatatum (Vasellati, 2001) and Genipa americana seedlings (Mielke, 2003), tomato (Walter, 2004); all of these plant species showed growth reduction to varying extents in waterlogged conditions

\section{Leaf number and leaf area}

Number of leaves was counted at each 7 days intervals after water logging treatment and was depicted in Table no 2, which showed that leaf number were decreased significantly in stressed plants than control as the duration of stress proceeds. Maximum number of leaf number was decreased in U.Pragyan followed by U.Pallavi at the rate of $26.4 \%$ and $17.2 \%$ respectively. The higher number of leaves was found in U.Kumari (69.6 leaves per plant and 64.7 leaves per plant) under 1 and 2 days stress condition respectively. However, the interaction was not found significant among the treatments and cultivars. Reduction in aerobic leaf area recorded at each 7 days interval after water logging was presented in Table no -1, showing the remarkable difference over control plants and was significant among the treatment and variety but individually under root hypoxia (Kreuzwieser et al., 2001). However there were variation within the four varieties but the U.Kumari was maximum leaf area of 9.5and $8.2 \mathrm{~cm} 2$ (under 1 day and 2 days of water logging respectively) after 23 days of its water logging, whereas U.Pragyan was minimum with 4.4 and $2.9 \mathrm{~cm} 2$ under 1 day and 2 days of water logging after 23 days. 
The cultivar U.Kumari was least leaf area decrease percent of $34.62 \%, 33.45 \%, 33.5 \%$, $33.55 \%$ under $2,9,16$ and 23 days of water logging respectively. The maximum reduction in leaf area was recorded in U.Pragyan $(59.96 \%)$ followed by U.Dipti $(50.39 \%)$ under two days of stagnant water. The changes in leaf number leaf size and leaf area indicated the short term water logging effect on the growth of plant parts than any other environmental factor (Walter 2004, Horchani F. et al., 2008).

\section{Shoot and root dry weight (g)}

Shoot dry weight was decreased in water logging condition than control in all the treatments and was presented in Table no 7 . Highest shoot dry weight was recorded in U.Kumari $(10.3 \mathrm{~g}, 8.9 \mathrm{~g}, 7.0 \mathrm{~g})$ followed by U.Pallavi $(9.4 \mathrm{~g}, 7.4 \mathrm{~g}, 6.2 \mathrm{~g})$ at a tune of $13.5 \%$ and $32 \%$ decrease over control in U.Kumari where as $21.2 \%$ and $34 \%$ decrease in U.Pallavi under both 1 and 2 days of water logging respectively. Similar trend was also recorded in root dry weight presented in Table no -11 with a decrease rate of 3.9 and $7.8 \%$ in 1 and 2 days of stagnant water over control in U.Kumari. Whereas the maximum reduction in root dry weight was observed in U. Dipti at a tune of $12.2 \%$ under 2 days of water logging. Whereas U.Kumari was minimum reduction in root dry weight of 3.9 and $7.8 \%$ under 1 and 2 days of water logging respectively. The drastic impact of hypoxia not only affect the roots but also limits the aerobic respiration and dramatically decrease the metabolism in aerial parts (Mommer et al., 2004).

\section{Total biomass $(\mathrm{g})$}

The total biomass of the experimental treatment wise data depicted in Table no-7 indicated that maximum biomass obtained in U.Kumari followed by U.Pallavi and U.Dipti and was decreased in treated plants than the control with a range of 26.7 to $59.3 \%$ in U.Kumari and U.Pallavi respectively. U.
Pragyan was very low total bio mass the significant responses within the variety treatment and their interaction was recorded among the treatments. The decrease in dry matter accumulation in response to low oxygen is a common response found in many plant species (Henshaw et al., 2007) and was suggested to be directly related to changes in net carbon assimilation that are attributed to limitation of photosynthesis(Pazeshki 2001).

\section{Chlorophyll and carotenoid content}

The pigments like chlorophyll, carotenoid content in leaves analyzed during the experimentation was presented in Table- 5 and 6 respectively indicated that the pigments synthesized during vegetative stage (chlorophyll and carotenoid) was decreased in the treated plants than the control from the days of withdrawal of stress up to 23 days at a tune of 8.5 to $27.4 \%$ in case of total chlorophyll and for carotenoid 3.6 to $17.5 \%$. The chlorophyll a was higher than chlorophyll $b$ but the percentage decrease of chlorophyll $b$ is more than the chlorophyll a (Horchani F. et al., 2008). U.Kumari was recorded with a less percentage decrease in both chlorophyll and carotenoid content of $8.5 \%$ and $3.6 \%$ under 1 day of stagnant water respectively.

Chlorophyll in Spad value was also in the same trend found significant in all treatments but was not significant in their interactions and presented in Table-4Conaty et al., (2008) reported the potentiality of variation in SPAD reading to differentiate the tolerant cotton cultivars under waterlogging. In relation to that carotenoid content also followed the similar trend of chlorophyll but was less in concentration $(\mathrm{mg} / \mathrm{g} F W)$ than the total chlorophyll and varies from 0.22 to $0.37 \mathrm{mg} / \mathrm{g}$ FW. The variety U.Kumari was the maximum concentration of concentration of chlorophyll and carotenoid followed by U.pallavi and U.dipti in all the date of observations. 
Table.1 Effect of water logging on plant height $(\mathrm{cm})$

\begin{tabular}{|c|c|c|c|c|c|c|c|c|c|c|c|c|}
\hline \multirow{2}{*}{$\begin{array}{l}\text { Plant height } \\
\text { (cm) } \\
\text { Variety }\end{array}$} & \multicolumn{3}{|c|}{2 DAYS AFTER WL } & \multicolumn{3}{|c|}{9 DAYS AFTER WL } & \multicolumn{3}{|c|}{16 DAYS AFTER WL } & \multicolumn{3}{|c|}{23 DAYS AFTER WL } \\
\hline & $\mathrm{S}_{\mathrm{O}}$ & $\mathrm{S}_{1}$ & $\mathrm{~S}_{2}$ & $\mathrm{~S}_{\mathrm{O}}$ & $\mathrm{S}_{1}$ & $\mathrm{~S}_{2}$ & $\mathrm{~S}_{\mathrm{O}}$ & $\mathrm{S}_{1}$ & $\mathrm{~S}_{2}$ & $\mathrm{~S}_{\mathrm{O}}$ & $\mathrm{S}_{1}$ & $\mathbf{S}_{2}$ \\
\hline U.Pallavi & 41.0 & $\begin{array}{l}37.4 \\
(8.7)\end{array}$ & $\begin{array}{l}34.6 \\
(15.5)\end{array}$ & 47.2 & $\begin{array}{l}42.7 \\
(9.7)\end{array}$ & $\begin{array}{l}38.2 \\
(19.1)\end{array}$ & 53.5 & $\begin{array}{l}47.7 \\
(10.8)\end{array}$ & $\begin{array}{l}42.9 \\
(19.9)\end{array}$ & 62.8 & $\begin{array}{l}53.8 \\
(14.3)\end{array}$ & $\begin{array}{l}50.2 \\
(20.1)\end{array}$ \\
\hline U.Dipti & 37.8 & $\begin{array}{l}34.7 \\
(8.2)\end{array}$ & $\begin{array}{l}33.2 \\
(12.2)\end{array}$ & 43.1 & $\begin{array}{l}41.2 \\
(4.5)\end{array}$ & $\begin{array}{l}40.0 \\
(7.2)\end{array}$ & 49.6 & $\begin{array}{l}43.9 \\
(11.6)\end{array}$ & $\begin{array}{l}42.3 \\
(14.7)\end{array}$ & 60.1 & $\begin{array}{l}46.9 \\
(22.0)\end{array}$ & $\begin{array}{l}46.2 \\
(23.1)\end{array}$ \\
\hline U.Kumari & 40.5 & $\begin{array}{l}37.7 \\
(6.9)\end{array}$ & $\begin{array}{l}34.5 \\
(14.8)\end{array}$ & 48.0 & $\begin{array}{l}41.8 \\
(12.9)\end{array}$ & $\begin{array}{l}37.3 \\
(23.2)\end{array}$ & 53.1 & $\begin{array}{l}46.0 \\
(13.4)\end{array}$ & $\begin{array}{l}40.3 \\
(24.0)\end{array}$ & 63.2 & $\begin{array}{l}53.5 \\
(15.3)\end{array}$ & $\begin{array}{l}51.2 \\
(18.9)\end{array}$ \\
\hline U.Pragyan & 29.1 & $\begin{array}{l}27.6 \\
(5.3)\end{array}$ & $\begin{array}{l}23.7 \\
(18.7)\end{array}$ & 36.4 & $\begin{array}{l}31.8 \\
(12.6)\end{array}$ & $\begin{array}{l}26.8 \\
(26.4)\end{array}$ & 42.4 & $\begin{array}{l}36.0 \\
(15.1)\end{array}$ & $\begin{array}{l}33.9 \\
(19.9)\end{array}$ & 51.5 & $\begin{array}{l}42.7 \\
(17.2)\end{array}$ & $\begin{array}{l}40.3 \\
(21.7)\end{array}$ \\
\hline & V & $\mathrm{S}$ & $V \times S$ & $\mathrm{~V}$ & $\mathrm{~S}$ & $\mathrm{~V} \times \mathrm{S}$ & $\mathrm{V}$ & $\mathrm{S}$ & $\mathrm{V} \times \mathrm{S}$ & $\mathrm{V}$ & S & $\mathbf{V} \times S$ \\
\hline $\mathrm{SE}(\mathrm{m}) \pm$ & 0.619 & 0.536 & 1.072 & 0.71 & 0.614 & 1.229 & 0.66 & 0.750 & 1.499 & 0.717 & 0.621 & 1.242 \\
\hline CD 5\% & 1.806 & 1.564 & 3.28 & 2.071 & 1.792 & 3.587 & 1.980 & 2.188 & 4.375 & 2.093 & 1.812 & 3.624 \\
\hline $\mathrm{CV} \%$ & 7.47 & & & 7.40 & & & 8.82 & & & 6.71 & & \\
\hline
\end{tabular}

S0-Control , S1-1day of water logging and S2- 2 days of waterlogging

Data presented as mean value of 3replications

Figure in parentheses indicates \% decrease over control 
Table.2 Effect of water logging on the number of leaves per plant

\begin{tabular}{|c|c|c|c|c|c|c|c|c|c|c|c|c|}
\hline \multirow{2}{*}{$\begin{array}{c}\text { LEAF NO. } \\
\text { Variety }\end{array}$} & \multicolumn{3}{|c|}{2 DAYS AFTER WL } & \multicolumn{3}{|c|}{9 DAYS AFTER WL } & \multicolumn{3}{|c|}{16 DAYS AFTER WL } & \multicolumn{3}{|c|}{23 DAYS AFTER WL } \\
\hline & $\mathbf{S}_{\mathbf{O}}$ & $\mathbf{S}_{\mathbf{1}}$ & $\mathbf{S}_{2}$ & $\mathbf{S}_{\mathbf{O}}$ & $\mathbf{S}_{1}$ & $\mathbf{S}_{\mathbf{2}}$ & $\mathbf{S}_{\mathbf{O}}$ & $\mathbf{S}_{1}$ & $\mathbf{S}_{\mathbf{2}}$ & $\mathbf{S}_{\mathbf{O}}$ & $\mathbf{S}_{1}$ & $\mathbf{S}_{2}$ \\
\hline U.Pallavi & 34.3 & $\begin{array}{l}31.3 \\
(8.7)\end{array}$ & $\begin{array}{l}29.3 \\
(14.6)\end{array}$ & 46.0 & $\begin{array}{l}41.0 \\
(10.9)\end{array}$ & $\begin{array}{l}35.7 \\
(22.5)\end{array}$ & 55.0 & $\begin{array}{l}50.0 \\
(9.1)\end{array}$ & $\begin{array}{l}44.7 \\
(18.8)\end{array}$ & 67.6 & $\begin{array}{l}61.3 \\
(14.3)\end{array}$ & $\begin{array}{l}56.0 \\
(17.2)\end{array}$ \\
\hline U.Dipti & 32.6 & $\begin{array}{l}27.6 \\
(15.3)\end{array}$ & $\begin{array}{l}26.6 \\
(18.4)\end{array}$ & 43.3 & $\begin{array}{l}37.6 \\
(13.1)\end{array}$ & $\begin{array}{l}33.7 \\
(22.3)\end{array}$ & 54.3 & $\begin{array}{l}48.3 \\
(11.0)\end{array}$ & $\begin{array}{l}49.3 \\
(9.2)\end{array}$ & 65.7 & $\begin{array}{l}57.0 \\
(13.2)\end{array}$ & $\begin{array}{l}55.0 \\
(16.3)\end{array}$ \\
\hline U.Kumari & 42.3 & $\begin{array}{l}39.3 \\
(7.1)\end{array}$ & $\begin{array}{l}36.8 \\
(13.1)\end{array}$ & 54.3 & $\begin{array}{l}48.0 \\
(11.7)\end{array}$ & $\begin{array}{l}42.3 \\
(22.1)\end{array}$ & 64.0 & $\begin{array}{l}58.3 \\
(8.9)\end{array}$ & $\begin{array}{l}55.3 \\
(13.5)\end{array}$ & 77.6 & $\begin{array}{l}69.6 \\
(10.3)\end{array}$ & $\begin{array}{l}64.7 \\
(16.7)\end{array}$ \\
\hline U.Pragyan & 29.8 & $\begin{array}{l}25.3 \\
(15.0)\end{array}$ & $\begin{array}{l}23.6 \\
(20.6)\end{array}$ & 40 & $\begin{array}{l}34.3 \\
(14.6)\end{array}$ & $\begin{array}{l}29.0 \\
(27.9)\end{array}$ & 48.3 & $\begin{array}{l}42.0 \\
(12.5)\end{array}$ & $\begin{array}{l}36.0 \\
(25.0)\end{array}$ & 58.6 & $\begin{array}{l}52.6 \\
(11.9)\end{array}$ & $\begin{array}{l}44.0 \\
(26.4)\end{array}$ \\
\hline & $\mathbf{V}$ & $\mathbf{S}$ & $\mathbf{V} \times \mathbf{S}$ & $\mathbf{V}$ & $\mathbf{S}$ & $\mathbf{V} \times S$ & $\mathbf{V}$ & $\mathbf{S}$ & $\mathbf{V} \times S$ & $\mathbf{V}$ & $\mathbf{S}$ & $\mathbf{V} \times \mathbf{S}$ \\
\hline $\mathrm{SE}(\mathrm{m}) \pm$ & 0.940 & 0.814 & NS & 0.940 & 0.814 & NS & 0.521 & 0.451 & NS & 0.611 & 0.529 & 1.058 \\
\hline CD $5 \%$ & 2.742 & 2.375 & NS & 2.742 & 2.375 & NS & 1.521 & 1.317 & NS & 1.784 & 1.545 & 3.089 \\
\hline $\mathrm{CV} \%$ & 9.20 & & & 9.20 & & & 3.88 & & & 3.63 & & \\
\hline
\end{tabular}

S0-Control, S1-1day of water logging and S2- 2 days of waterlogging

Data presented as mean value of 3 replications

Figure in parentheses indicates \% decrease overcontrol 
Table.3 Effect of water logging on the leaf area $\left(\mathrm{cm}^{2}\right)$

\begin{tabular}{|c|c|c|c|c|c|c|c|c|c|c|c|c|}
\hline \multirow{2}{*}{$\begin{array}{l}\text { Leaf area } \\
\left(\mathrm{cm}^{2}\right)\end{array}$} & \multicolumn{3}{|c|}{2 DAYS AFTER WL } & \multicolumn{3}{|c|}{9 DAYS AFTER WL } & \multicolumn{3}{|c|}{16 DAYS AFTER WL } & \multicolumn{3}{|c|}{23 DAYS AFTER WL } \\
\hline & $\mathbf{S}_{\mathbf{O}}$ & $\mathbf{S}_{1}$ & $\mathbf{S}_{2}$ & $\mathbf{S}_{\mathbf{O}}$ & $\mathbf{S}_{1}$ & $\mathbf{S}_{2}$ & $\mathbf{S}_{\mathbf{o}}$ & $\mathbf{S}_{1}$ & $\mathbf{S}_{2}$ & $\mathbf{S}_{\mathbf{O}}$ & $\mathbf{S}_{1}$ & $\mathbf{S}_{\mathbf{2}}$ \\
\hline U.Pallavi & 6.9 & $\begin{array}{c}5.8 \\
(15.3)\end{array}$ & $\begin{array}{c}4.5 \\
(34.7)\end{array}$ & 8.1 & $\begin{array}{c}6.3 \\
(22.9)\end{array}$ & $\begin{array}{c}5.1 \\
(37.1)\end{array}$ & 8.9 & $\begin{array}{c}6.5 \\
(26.8)\end{array}$ & $\begin{array}{c}5.5 \\
(38.2)\end{array}$ & 8.9 & $\begin{array}{c}6.5 \\
(26.8)\end{array}$ & $\begin{array}{c}5.5 \\
(38.2)\end{array}$ \\
\hline U.Dipti & 8.0 & $\begin{array}{c}5.8 \\
(27.3)\end{array}$ & $\begin{array}{c}4.1 \\
(48.5)\end{array}$ & 9.7 & $\begin{array}{c}6.8 \\
(30.4)\end{array}$ & $\begin{array}{c}4.7 \\
(51.5)\end{array}$ & 10.3 & $\begin{array}{c}7.0 \\
(31.5)\end{array}$ & $\begin{array}{c}5.1 \\
(50.3)\end{array}$ & 10.3 & $\begin{array}{c}7.0 \\
(31.5)\end{array}$ & $\begin{array}{c}5.1 \\
(50.3)\end{array}$ \\
\hline U.Kumari & 10.1 & $\begin{array}{c}7.8 \\
(22.6)\end{array}$ & $\begin{array}{c}6.6 \\
(34.6)\end{array}$ & 12.0 & $\begin{array}{c}9.2 \\
(23.2)\end{array}$ & $\begin{array}{c}8.0 \\
(33.4)\end{array}$ & 12.3 & $\begin{array}{c}9.5 \\
(22.9)\end{array}$ & $\begin{array}{c}8.2 \\
(33.5)\end{array}$ & 12.3 & $\begin{array}{c}9.5 \\
(22.9)\end{array}$ & $\begin{array}{c}8.2 \\
(33.5)\end{array}$ \\
\hline \multirow[t]{2}{*}{ U.Pragyan } & 5.8 & $\begin{array}{c}3.7 \\
(37.4)\end{array}$ & $\begin{array}{c}2.5 \\
(56.5)\end{array}$ & 6.8 & $\begin{array}{c}4.0 \\
(41.1)\end{array}$ & $\begin{array}{c}2.9 \\
(57.2)\end{array}$ & 7.2 & $\begin{array}{c}4.4 \\
(39.9)\end{array}$ & $\begin{array}{c}2.9 \\
(59.9)\end{array}$ & 7.2 & $\begin{array}{c}4.4 \\
(39.9)\end{array}$ & $\begin{array}{c}2.9 \\
(59.9)\end{array}$ \\
\hline & $\mathbf{V}$ & $\mathbf{S}$ & $\mathbf{V} \times S$ & $\mathbf{V}$ & $\mathbf{S}$ & $\mathbf{V} \times S$ & $\mathbf{V}$ & $\mathbf{S}$ & $\mathbf{V} \times S$ & $\mathbf{V}$ & $\mathbf{S}$ & $\mathbf{V} \times S$ \\
\hline $\mathrm{SE}(\mathrm{m}) \pm$ & 0.240 & 0.208 & NS & 0.197 & 0.170 & NS & 0.287 & 0.248 & NS & 0.287 & 0.248 & NS \\
\hline CD $5 \%$ & 0.700 & 0.607 & NS & 0.574 & 0.497 & NS & 0.837 & 0.725 & NS & 0.837 & 0.725 & NS \\
\hline CV \% & 12.07 & & & 8.47 & & & 11.75 & & & 11.75 & & \\
\hline
\end{tabular}

S0-Control , S1-1day of water logging and S2- 2 days of waterlogging

Data presented as mean value of 3replications

Figure in parentheses indicates $\%$ decrease overcontrol 
Table.4 SPAD Values of chlorophyll content in leaves in response to water logging

\begin{tabular}{|c|c|c|c|c|c|c|c|c|c|c|c|c|}
\hline $\begin{array}{l}\text { SPAD } \\
\text { Value }\end{array}$ & \multicolumn{3}{|c|}{2 DAYS AFTER WL } & \multicolumn{3}{|c|}{9 DAYS AFTER WL } & \multicolumn{3}{|c|}{16 DAYS AFTER WL } & \multicolumn{3}{|c|}{23 DAYS AFTER WL } \\
\hline Variety & $\mathbf{S}_{\mathbf{O}}$ & $\mathbf{S}_{1}$ & $\mathbf{S}_{\mathbf{2}}$ & $\mathbf{S}_{\mathbf{O}}$ & $\mathbf{S}_{1}$ & $\mathbf{S}_{2}$ & $\mathbf{S}_{\mathbf{O}}$ & $\mathbf{S}_{1}$ & $\mathbf{S}_{2}$ & $\mathbf{S}_{\mathbf{O}}$ & $\mathbf{S}_{1}$ & $\mathbf{S}_{2}$ \\
\hline U.Pallavi & 8.5 & $\begin{array}{c}7.3 \\
(14.1)\end{array}$ & $\begin{array}{c}6.2 \\
(27.1)\end{array}$ & 9.2 & $\begin{array}{c}7.5 \\
(18.5)\end{array}$ & $\begin{array}{c}6.6 \\
(28.3)\end{array}$ & 9.5 & $\begin{array}{c}7.7 \\
(18.9)\end{array}$ & $\begin{array}{c}6.8 \\
(28.4)\end{array}$ & 9.6 & $\begin{array}{c}7.8 \\
(18.8)\end{array}$ & $\begin{array}{c}6.9 \\
(28.1)\end{array}$ \\
\hline U.Dipti & 8.2 & $\begin{array}{c}7.4 \\
(9.8)\end{array}$ & $\begin{array}{c}6.4 \\
(22.0)\end{array}$ & 8.5 & $\begin{array}{c}7.8 \\
(8.2)\end{array}$ & $\begin{array}{c}6.9 \\
(18.8)\end{array}$ & 8.9 & $\begin{array}{c}8.1 \\
(9.0)\end{array}$ & $\begin{array}{c}7 \\
(21.3)\end{array}$ & 9 & $\begin{array}{c}8.2 \\
(8.9)\end{array}$ & $\begin{array}{c}7.1 \\
(21.1)\end{array}$ \\
\hline U.Kumari & 8.9 & $\begin{array}{c}8.1 \\
(9.0)\end{array}$ & $\begin{array}{c}7.2 \\
(19.1)\end{array}$ & 9.5 & $\begin{array}{c}8.6 \\
(9.5)\end{array}$ & $\begin{array}{c}7.8 \\
(17.9)\end{array}$ & 9.8 & $\begin{array}{c}9 \\
(8.2)\end{array}$ & $\begin{array}{c}8.1 \\
(17.3)\end{array}$ & 9.9 & $\begin{array}{c}9.3 \\
(6.1)\end{array}$ & $\begin{array}{c}8.2 \\
(17.2)\end{array}$ \\
\hline U.Pragyan & 8.2 & $\begin{array}{c}7.1 \\
(13.4)\end{array}$ & $\begin{array}{c}6 \\
(26.8)\end{array}$ & 8.6 & $\begin{array}{c}7.4 \\
(14.0)\end{array}$ & $\begin{array}{c}6.4 \\
(25.6)\end{array}$ & 8.8 & $\begin{array}{c}7.5 \\
(14.8)\end{array}$ & $\begin{array}{c}6.5 \\
(26.1)\end{array}$ & 8.9 & $\begin{array}{c}7.6 \\
(14.6)\end{array}$ & $\begin{array}{c}6.6 \\
(25.8)\end{array}$ \\
\hline & $\mathbf{V}$ & $\mathbf{S}$ & $\mathbf{V} \times \mathbf{S}$ & $\mathbf{V}$ & $\mathbf{S}$ & $\mathbf{V} \times \mathbf{S}$ & $\mathbf{V}$ & $\mathbf{S}$ & $\mathbf{V} \times \mathbf{S}$ & $\mathbf{V}$ & $\mathbf{S}$ & $\mathbf{V} \times \mathbf{S}$ \\
\hline $\mathrm{SE}(\mathrm{m}) \pm$ & NS & 0.375 & NS & NS & 0.404 & NS & NS & 0.375 & NS & NS & 0.346 & NS \\
\hline CD $5 \%$ & NS & 1.095 & NS & NS & 1.179 & NS & NS & 1.095 & NS & NS & 1.011 & NS \\
\hline CV \% & 17.43 & & & 17.72 & & & 15.97 & & & 14.53 & & \\
\hline
\end{tabular}

S0-Control, S1-1day of water logging and S2- 2 days of waterlogging

Figure in parenthesis indicates \% decrease overcontrol

Data presented as mean value of 3replications 
Table.5 Total chlorophyll content (mg/g FW) in response to water logging

\begin{tabular}{|c|c|c|c|c|c|c|c|c|c|c|c|c|}
\hline \multirow{2}{*}{$\begin{array}{l}\text { Tot. } \\
\text { Chlorophyll } \\
\text { (Mg/g FW) }\end{array}$} & \multicolumn{3}{|c|}{2 DAYS AFTER WL } & \multicolumn{3}{|c|}{9 DAYS AFTER WL } & \multicolumn{3}{|c|}{16 DAYS AFTER WL } & \multicolumn{3}{|c|}{23 DAYS AFTER WL } \\
\hline & $\mathbf{S}_{\mathbf{O}}$ & $\mathbf{S}_{1}$ & $\mathbf{S}_{\mathbf{2}}$ & $\mathbf{S}_{\mathbf{O}}$ & $\mathbf{S}_{1}$ & $\mathbf{S}_{\mathbf{2}}$ & $\mathbf{S}_{\mathbf{O}}$ & $\mathbf{S}_{1}$ & $\mathbf{S}_{\mathbf{2}}$ & $\mathbf{S}_{\mathbf{O}}$ & $\mathbf{S}_{1}$ & $\mathbf{S}_{\mathbf{2}}$ \\
\hline U.Pallavi & 1.14 & $\begin{array}{l}1.01 \\
(11.4)\end{array}$ & $\begin{array}{l}0.97 \\
(14.9)\end{array}$ & 1.34 & $\begin{array}{l}1.03 \\
(23.5)\end{array}$ & $\begin{array}{l}0.99 \\
(25.5)\end{array}$ & 1.36 & $\begin{array}{l}1.04 \\
(23.5)\end{array}$ & $\begin{array}{l}0.98 \\
(27.9)\end{array}$ & 1.35 & $\begin{array}{l}1.05 \\
(23.0)\end{array}$ & $\begin{array}{l}0.98 \\
(27.4)\end{array}$ \\
\hline U.Dipti & 1.1 & $\begin{array}{l}0.96 \\
(12.7)\end{array}$ & $\begin{array}{l}0.92 \\
(16.4)\end{array}$ & 1.29 & $\begin{array}{l}0.98 \\
(24.7)\end{array}$ & $\begin{array}{l}0.94 \\
(25.8)\end{array}$ & 1.31 & $\begin{array}{c}1 \\
(23.7)\end{array}$ & $\begin{array}{l}0.96 \\
(26.7)\end{array}$ & 1.31 & $\begin{array}{l}1.01 \\
(23.7)\end{array}$ & $\begin{array}{l}0.96 \\
(26.7)\end{array}$ \\
\hline U.Kumari & 1.24 & $\begin{array}{l}1.21 \\
(2.4)\end{array}$ & $\begin{array}{l}1.13 \\
(8.9)\end{array}$ & 1.36 & $\begin{array}{l}1.22 \\
(9.2)\end{array}$ & $\begin{array}{l}1.17 \\
(13.3)\end{array}$ & 1.39 & $\begin{array}{l}1.26 \\
(9.4)\end{array}$ & $\begin{array}{c}1.2 \\
(13.7)\end{array}$ & 1.41 & $\begin{array}{l}1.29 \\
(8.5)\end{array}$ & $\begin{array}{l}1.22 \\
(13.5)\end{array}$ \\
\hline U.Pragyan & 1.14 & $\begin{array}{l}1.03 \\
(9.6)\end{array}$ & $\begin{array}{l}0.94 \\
(17.5)\end{array}$ & 1.2 & $\begin{array}{l}1.05 \\
(10.1)\end{array}$ & $\begin{array}{l}0.96 \\
(16.9)\end{array}$ & 1.23 & $\begin{array}{l}1.07 \\
(13.0)\end{array}$ & $\begin{array}{l}0.97 \\
(21.1)\end{array}$ & 1.23 & $\begin{array}{l}1.07 \\
(13.0)\end{array}$ & $\begin{array}{l}0.97 \\
(21.1)\end{array}$ \\
\hline & $\mathbf{V}$ & $\mathbf{S}$ & $\mathbf{V} \times \mathbf{S}$ & $\mathbf{V}$ & $\mathbf{S}$ & $\mathbf{V} \times \mathbf{S}$ & $\mathbf{V}$ & $\mathbf{S}$ & $\mathbf{V} \times \mathbf{S}$ & $\mathbf{V}$ & $\mathbf{S}$ & $\mathbf{V} \times \mathbf{S}$ \\
\hline $\mathrm{SE}(\mathrm{m}) \pm$ & 0.003 & 0.003 & 0.006 & 0.013 & 0.012 & 0.023 & 0.020 & 0.017 & NS & 0.014 & 0.012 & 0.025 \\
\hline CD $5 \%$ & 0.010 & 0.008 & 0.017 & 0.039 & 0.034 & 0.067 & 0.058 & 0.051 & NS & 0.042 & 0.036 & 0.072 \\
\hline CV \% & 0.94 & & & 3.55 & & & 5.23 & & & 3.73 & & \\
\hline
\end{tabular}

S0-Control , S1-1day of water logging and S2- 2 days of waterlogging

Figure in parenthesis indicates \% decrease overcontrol

Data presented as mean value of 3replications 
Table.6 Effect of water logging on carotenoid content (mg/g FW)

\begin{tabular}{|c|c|c|c|c|c|c|c|c|c|c|c|c|}
\hline \multirow{2}{*}{$\begin{array}{r}\text { Carotenoid } \\
(\mathrm{mg} / \mathrm{g} \text { FW) }\end{array}$} & \multicolumn{3}{|c|}{2 DAYS AFTER WL } & \multicolumn{3}{|c|}{9 DAYS AFTER WL } & \multicolumn{3}{|c|}{16 DAYS AFTER WL } & \multicolumn{3}{|c|}{23 DAYS AFTER WL } \\
\hline & $\mathbf{S}_{\mathbf{O}}$ & $\mathbf{S}_{1}$ & $\mathbf{S}_{\mathbf{2}}$ & $\mathbf{S}_{\mathbf{O}}$ & $\mathbf{S}_{1}$ & $\mathbf{S}_{\mathbf{2}}$ & $\mathbf{S}_{\mathbf{O}}$ & $\mathbf{S}_{1}$ & $\mathbf{S}_{\mathbf{2}}$ & $\mathbf{S}_{\mathbf{O}}$ & $\mathbf{S}_{1}$ & $\mathbf{S}_{\mathbf{2}}$ \\
\hline U.Pallavi & 0.52 & $\begin{array}{l}0.48 \\
(7.7)\end{array}$ & $\begin{array}{l}0.44 \\
(15.4)\end{array}$ & 0.56 & $\begin{array}{l}0.49 \\
(12.5)\end{array}$ & $\begin{array}{l}0.46 \\
(17.9)\end{array}$ & 0.57 & $\begin{array}{l}0.49 \\
(14.0)\end{array}$ & $\begin{array}{l}0.47 \\
(17.5)\end{array}$ & 0.57 & $\begin{array}{c}0.5 \\
(12.3)\end{array}$ & $\begin{array}{l}0.47 \\
(17.5)\end{array}$ \\
\hline U.Dipti & 0.49 & $\begin{array}{l}0.45 \\
(8.2)\end{array}$ & $\begin{array}{l}0.43 \\
(12.2)\end{array}$ & 0.51 & $\begin{array}{l}0.47 \\
(7.8)\end{array}$ & $\begin{array}{l}0.44 \\
(13.7)\end{array}$ & 0.52 & $\begin{array}{l}0.48 \\
(7.7)\end{array}$ & $\begin{array}{l}0.46 \\
(11.5)\end{array}$ & 0.53 & $\begin{array}{l}0.48 \\
(9.4)\end{array}$ & $\begin{array}{l}0.47 \\
(11.3)\end{array}$ \\
\hline U.Kumari & 0.53 & $\begin{array}{l}0.51 \\
(3.8)\end{array}$ & $\begin{array}{c}0.5 \\
(5.7)\end{array}$ & 0.54 & $\begin{array}{l}0.52 \\
(3.7)\end{array}$ & $\begin{array}{l}0.51 \\
(5.6)\end{array}$ & 0.55 & $\begin{array}{l}0.53 \\
(3.6)\end{array}$ & $\begin{array}{l}0.51 \\
(7.3)\end{array}$ & 0.56 & $\begin{array}{l}0.54 \\
(3.6)\end{array}$ & $\begin{array}{l}0.52 \\
(7.1)\end{array}$ \\
\hline U.Pragyan & 0.5 & $\begin{array}{l}0.44 \\
(12.0)\end{array}$ & $\begin{array}{l}0.42 \\
(16.0)\end{array}$ & 0.51 & $\begin{array}{l}0.45 \\
(11.8)\end{array}$ & $\begin{array}{l}0.42 \\
(17.6)\end{array}$ & 0.51 & $\begin{array}{l}0.46 \\
(9.8)\end{array}$ & $\begin{array}{l}0.43 \\
(15.7)\end{array}$ & 0.53 & $\begin{array}{l}0.47 \\
(11.3)\end{array}$ & $\begin{array}{l}0.44 \\
(17.0)\end{array}$ \\
\hline & $\mathbf{V}$ & $\mathbf{S}$ & $\mathbf{V} \times S$ & $\mathbf{V}$ & $\mathbf{S}$ & $\mathbf{V} \times \mathbf{S}$ & V & $\mathbf{S}$ & $\mathbf{V} \times \mathbf{S}$ & V & $\mathbf{S}$ & $\mathbf{V} \times S$ \\
\hline $\mathrm{SE}(\mathrm{m}) \pm$ & 0.001 & 0.001 & 0.001 & 0.001 & 0.001 & 0.002 & 0.002 & 0.001 & 0.003 & 0.002 & 0.002 & 0.003 \\
\hline CD $5 \%$ & 0.002 & 0.002 & 0.003 & 0.003 & 0.003 & 0.005 & 0.005 & 0.004 & 0.008 & 0.006 & 0.005 & 0.010 \\
\hline $\mathrm{CV} \%$ & 0.62 & & & 1.04 & & & 1.74 & & & 1.83 & & \\
\hline
\end{tabular}

S0-Control , S1-1day of water logging and S2- 2 days of waterlogging

Parenthesis in the table indicates percent decrease over control

Data presented as mean value of 3replications 
Table.7 Dry weight of roots $\left(\mathrm{g}_{\text {plant }}{ }^{-1}\right)$, Dry weight of shoot $\left(\mathrm{g} \mathrm{plant}^{-1}\right)$ and Total biomass $\left(\mathrm{g} \mathrm{plant}^{-1}\right)$ in response to water logging

\begin{tabular}{|c|c|c|c|c|c|c|c|c|c|c|}
\hline \multicolumn{2}{|l|}{ Treatment } & \multicolumn{3}{|c|}{ DW root $(g)$} & \multicolumn{3}{|c|}{ Total biomass (g) } & \multicolumn{3}{|c|}{ DW shoot (g) } \\
\hline \multirow{3}{*}{ U.Pallavi } & So & \multicolumn{3}{|c|}{4.6} & \multicolumn{3}{|c|}{44.7} & \multicolumn{3}{|c|}{9.4} \\
\hline & S1 & \multicolumn{3}{|c|}{$\begin{array}{c}4.4 \\
(4.3)\end{array}$} & \multicolumn{3}{|c|}{$\begin{array}{l}30.8 \\
(31)\end{array}$} & \multicolumn{3}{|c|}{$\begin{array}{c}7.4 \\
(21.2)\end{array}$} \\
\hline & S2 & \multicolumn{3}{|c|}{$\begin{array}{c}4.1 \\
(10.8)\end{array}$} & \multicolumn{3}{|c|}{$\begin{array}{c}17.9 \\
(59.3)\end{array}$} & \multicolumn{3}{|c|}{$\begin{array}{c}6.2 \\
(34.0)\end{array}$} \\
\hline \multirow{3}{*}{ U.Dipti } & So & \multicolumn{3}{|c|}{4.9} & \multicolumn{3}{|c|}{41.1} & \multicolumn{3}{|c|}{9.2} \\
\hline & S1 & \multicolumn{3}{|c|}{$\begin{array}{c}4.6 \\
(6.1)\end{array}$} & \multicolumn{3}{|c|}{$\begin{array}{c}24.0 \\
(41.5)\end{array}$} & \multicolumn{3}{|c|}{$\begin{array}{c}7.2 \\
(21.7)\end{array}$} \\
\hline & $\mathbf{S 2}$ & \multicolumn{3}{|c|}{$\begin{array}{c}4.3 \\
(12.2)\end{array}$} & \multicolumn{3}{|c|}{$\begin{array}{c}19.1 \\
(53.5)\end{array}$} & \multicolumn{3}{|c|}{$\begin{array}{c}6.0 \\
(34.7)\end{array}$} \\
\hline \multirow{3}{*}{ U.Kumari } & So & \multicolumn{3}{|c|}{5.1} & \multicolumn{3}{|c|}{53.6} & \multicolumn{3}{|c|}{10.3} \\
\hline & S1 & \multicolumn{3}{|c|}{$\begin{array}{c}4.9 \\
(3.9)\end{array}$} & \multicolumn{3}{|c|}{$\begin{array}{c}39.3 \\
(26.7)\end{array}$} & \multicolumn{3}{|c|}{$\begin{array}{c}8.9 \\
(13.5)\end{array}$} \\
\hline & $\mathbf{S 2}$ & \multicolumn{3}{|c|}{$\begin{array}{c}4.7 \\
(7.8)\end{array}$} & \multicolumn{3}{|c|}{$\begin{array}{c}27.1 \\
(49.4)\end{array}$} & \multicolumn{3}{|c|}{$\begin{array}{c}7 \\
(32)\end{array}$} \\
\hline & So & \multicolumn{3}{|c|}{4.5} & & 21.1 & & & 7.7 & \\
\hline U.Pragyan & S1 & & $\begin{array}{c}4.3 \\
(4.4)\end{array}$ & & & $\begin{array}{c}13.6 \\
(35.7)\end{array}$ & & & $\begin{array}{c}6.1 \\
(20.7)\end{array}$ & \\
\hline & S2 & & $\begin{array}{c}4 \\
(11.1)\end{array}$ & & & $\begin{array}{c}9.8 \\
(53.8)\end{array}$ & & & $\begin{array}{c}5.2 \\
(32.4)\end{array}$ & \\
\hline & & $\mathrm{V}$ & $\mathrm{S}$ & $\mathrm{V} \times \mathrm{S}$ & $\mathrm{V}$ & $\mathrm{S}$ & $\mathrm{V} \times \mathrm{S}$ & $\mathrm{V}$ & $\mathrm{S}$ & $\mathbf{V} \times \mathbf{S}$ \\
\hline $\mathrm{SE}(\mathrm{m}) \pm$ & & 0.087 & 0.075 & $\mathrm{NS}$ & 0.131 & 0.114 & 0.228 & 0.141 & 0.122 & 0.224 \\
\hline CD 5\% & & 0.253 & 0.219 & NS & 0.384 & 0.332 & 0.664 & 0.411 & 0.356 & 0.711 \\
\hline CV \% & & 5.75 & & & 3.87 & & & 5.73 & & \\
\hline
\end{tabular}

S0-Control , S1-1day of water logging and S2- 2 days of waterlogging Parentheses in the table indicates percent decrease overcontrol.

Data presented as mean value of 3replications 


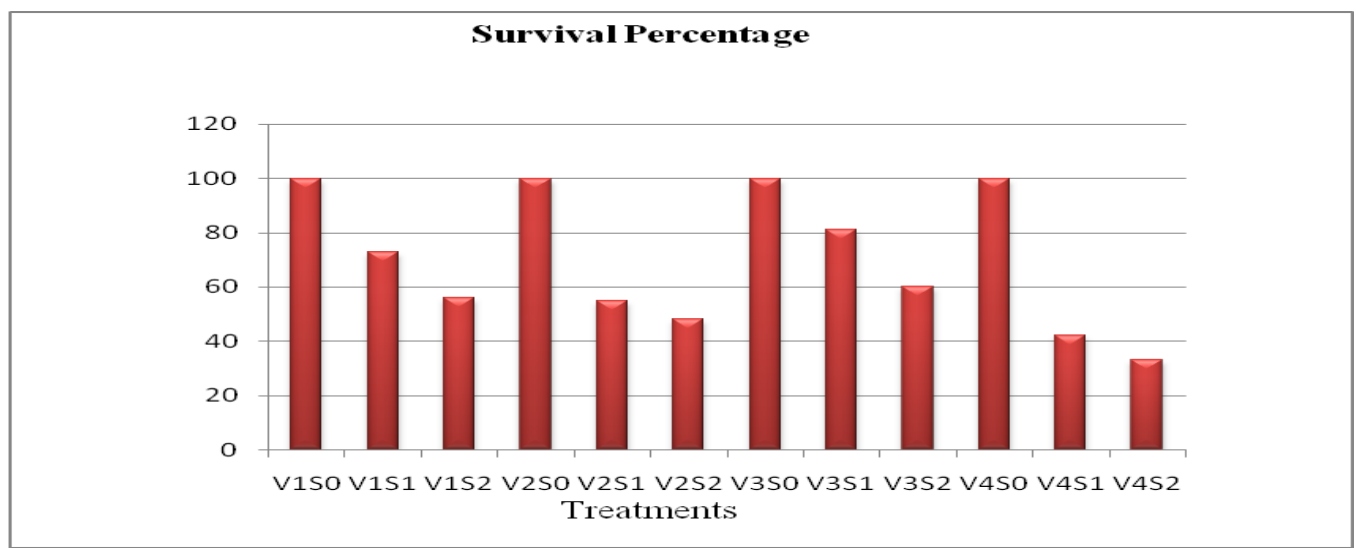

V1 - U. Pallavi, V2 - U. Dipti ,V3 - U. Kumari V4 - U. Pragyan

S0-Control ,S1-1day of water logging ,S2- 2days of water logging

** All plants were died under 3 days of water logging

Fig.1 Effect of water logging on survival percentage of tomato plant ( 2 days after recovery)

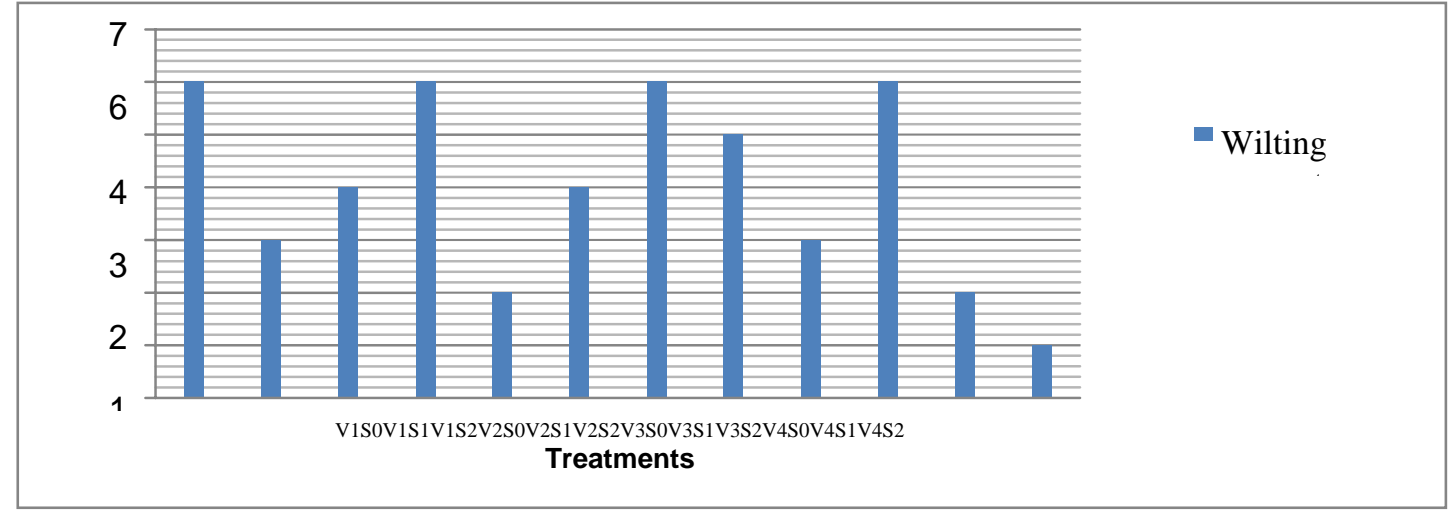

V1 - U. Pallavi, V2 - U. Dipti ,V3 - U. Kumari V4 - U.Pragyan S0-Control ,S1-1day of water logging ,S2- 2days of water logging ** All plants were died under 3 days of water logging

Fig. 2 Effect of water logging on wilting percentage of tomato plant(2 days after recovery)

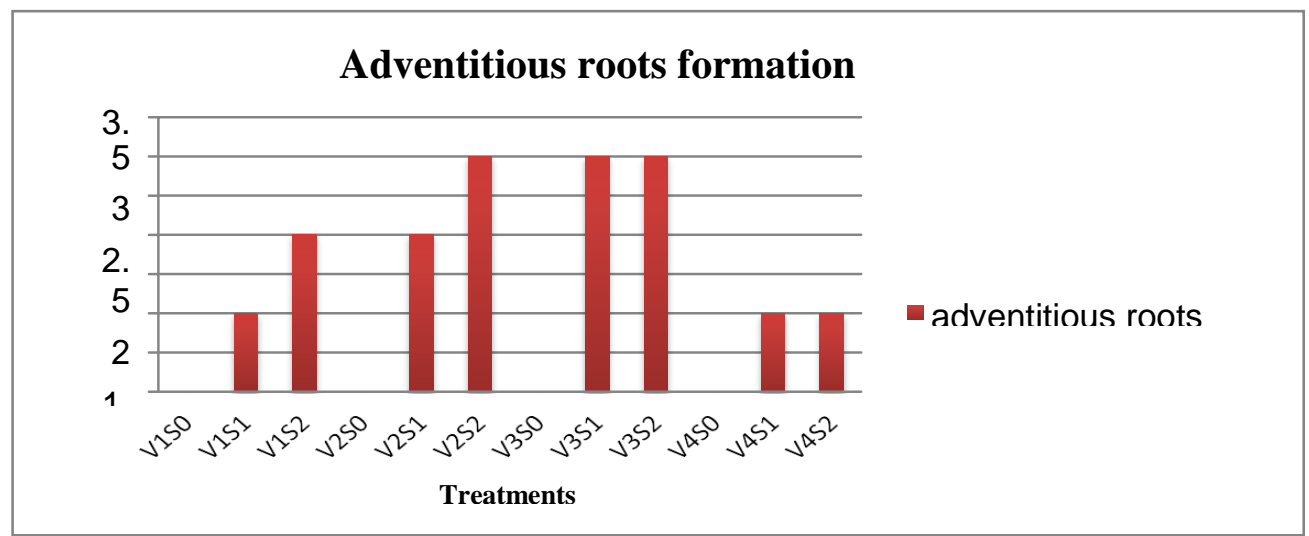

V1 - U. Pallavi, V2 - U. Dipti ,V3 - U. Kumari V4 - U.Pragyan S0-Control ,S1-1day of water logging ,S2- 2days of water logging ** All plants were died under 3 days of water logging

Fig.3 Adventitious root formation in response to water logging (2 days after recovery) 


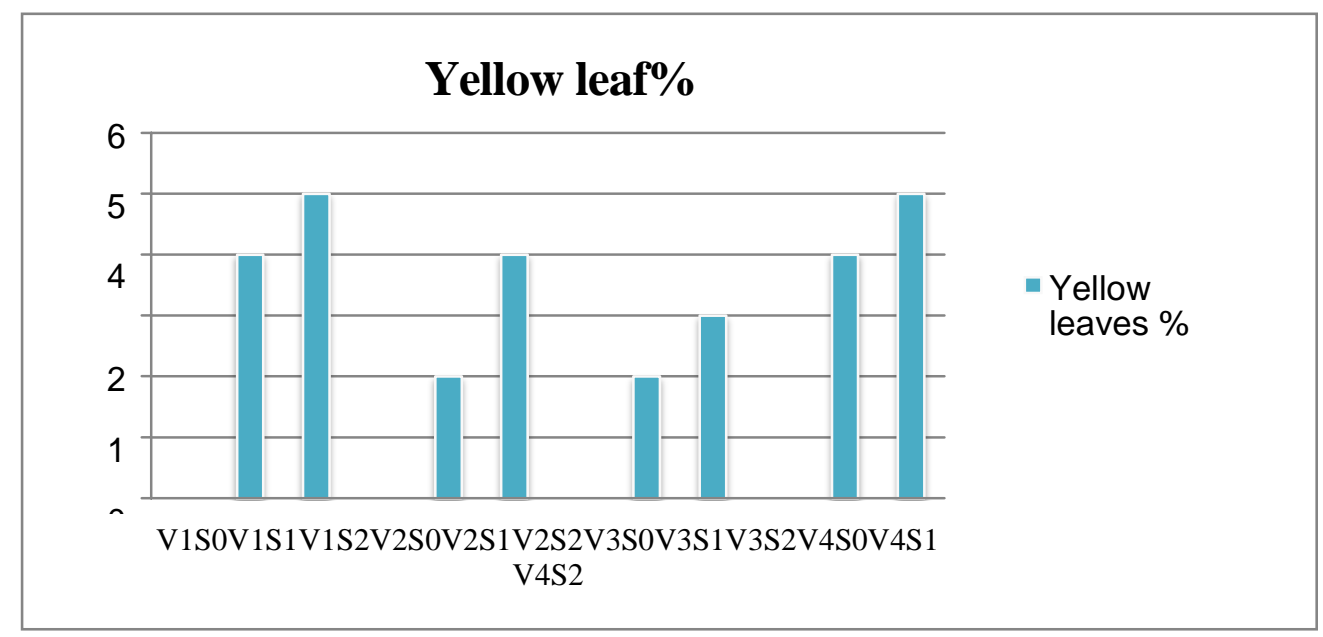

V1 - U. Pallavi, V2 - U. Dipti ,V3 - U. Kumari V4 - U.Pragyan S0-Control ,S1-1day of water logging ,S2-2days of water logging ** All plants were died under 3 days of water logging

Fig.4 Yellowing of leaves in response to water logging (2 days after recovery)

The consequences of hypoxia were not only confined to roots but also extended to aerial organs. Although all cultivars survived $15 \mathrm{~cm}$ of stagnant water for 2 days (all the plants died for 3 days of stagnant water in this experiment). Some symptoms of stress were observed. High survival percentage, Adventitious roots and low percentage of yellowing and wilting are some of the desirable characters for tolerating waterlogging as tomato is highly sensitive to excess water. U.Kumari was less affected under both one and two days of waterlogging as compared to other genotypes.

\section{References}

Aggarwal, P. K., Kalra, N., Chander, S. and Pathak H. 2006. InfoCrop: a dynamic simulation model for the assessment of cropyields, losses due to pests, and environmental impact of agro-ecosystems in tropical environments. I. Model description. Agric Syst. 89: 1-25.

Ahsana, N., Leea, D. G., Leea, S.H., Kanga, K.Y., Bahka, J.D., Choib, M. S., Leec, I. J., Renautd, J. and Leea, B. H. 2007. A comparative proteomic analysis of tomato leaves in response to water logging stress. Physiologia Plantarum. 131: 555-570.

Ashraf, M. 2003. Relationships between leaf gas exchange characteristics and growth of differently adapted populations of Blue panicgrass (Panicumantidotale Retz.) under salinity or waterlogging. Plant Sci.166: 69 - 75.

Bailey-Serres, J. and Voesenek, L.A.C.J. 2008. Flooding stress: acclimations and genetic diversity. Annu. Rev. Plant Biol. 59: 313-339.

Conaty, W.C., Tan, D.K.Y., Constable, G.A., Stton, B.G., Field, D. J. and Mamum, E. A. 2008. Genetic variation for waterlogging tolerance in cotton. $J$. Cotton Sci. 12: 53-61.

Dawood, T., Rieu, I., Wolters-Arts, M. D., Celestina, M. and Visser, E. J. W. 2013. Rapid flooding-induced adventitious root development from preformed primordia in Solanum dulcamara. AOB Plants.6:1-13.

Drew, MC. 1997. Oxygen deficiency and root metabolism: Injury and acclimation under hypoxia and anoxia. Ann. Rev. Plant Physiol. Plant Mol.Biol. 48:223250. 
Greenway, H. and Gibbs, J. 2003. Mechanisms of anoxia tolerance in plants. II. Energy requirements for maintenance and energy distribution to essential processes. Functional Plant Biology. 30(10).

Henshaw, T. L., Gilbert, R. A., Scholberg, J. M. S. and Sinclair, T. R. 2007a.Soya bean (Glycine max L. Merr.) genotype response to early-season flooding I. Root and nodule development. Agron.

Crop Sci.193:177-188.

Henshaw, T. L., Gilbert, R. A., Scholberg, J. M. S. and Sinclair, T. R. 2007b.Soya bean (Glycine $\max \mathrm{L}$ Merr.) genotype response to early-season flooding: II. Aboveground growth and biomass. Agron. Crop Sci. 193:189-197.

Horchani, F. and Smiti, A. S. 2010. Prolonged root hypoxia effect on enzymes involved in nitrogen assimilation pathway in tomato plants.Plant signalling \& Behavior.5(12): 15831589.

Horchani, F., Aloui, A., Brouquisse, R. and Smiti, A. S. 2008. Physiological Responses of Tomato Plants (Solanum lycopersicum) as affected by Root Hypoxia. Agronomy \& Crop Science.194: 297-303.

Horchani, F., Aloui, A., Brouquisse, R. and Smiti, A. S. 2008. Physiological Responses of Tomato Plants (Solanum lycopersicum) as affected by Root Hypoxia. Agronomy \& Crop Science. 194: 297-303.

Huang, B.R., Johnson, J. W., Nesmith, S. and Bridges, D.C. 1994a. Growth, physiological and anatomical responses of two wheat genotypes to waterlogging and nutrient supply. J. Exp. Bot. 45: 193-202.

Huang, B.R., Johnson, J.W., Nesmith, S. and Bridges, D.C. 1994b. Root and shoot growth of wheat genotypes in response to hypoxia and subsequent resumption of aeration. Crop Sci. 34:15381544.

Jackson, M.B. and Colmer, T.D. 2005. Response and adaptation by plants to flooding stress. Ann. Bot. 96: 501-505.

Kozlowski, T.T. 1997. Response of woody plants to flooding and salinity.Tree Physiology Monograf.1:1-29.

Kramer, P.J. 1951. Causes injury to plants resulting from flooding of the soil. Plant Physiol. 26:722-736.

Lin, K. H. R., Weng, C. C., Lo, H. F. and Chen, J. T. 2004. Study of the root antioxidative system of tomatoes and eggplants underwaterlogged conditions. Plant Sci. 167: 355- 365.

Mano, Y., Omori, F., Muraki, M. and Takamizo, T. 2005c. QTL mapping of adventitious root formation under flooding conditions in tropical maize (Zea mays L.) seedlings. Breed. Sci. 55: 343-347.

Mielke, M.S., Almeida, A.A.F., Gomes, F.P., Agular, M.A.F. and Mangabeira, P.A. O. 2003. Leaf gas exchange, chlorophyll fluorescence and growth responses of Genipa Americana seedlings to soil flooding. Environ. Exp. Bot. (article online):1-11.

Mommer, L., Pederson, O. and Visser, E. J. W. 2004. Acclimation of a terrestrial plant to submergence facilitates gas exchange under water. Plant Cell Environ. 27: 1281- 1287.

Nunez-Elisea, R., Schaffer, B., Fisher, J.B., Colls, A.M. and Crane, J.H. 1999. Influence of flooding on net $\mathrm{CO}_{2}$ assimilation, growth and stem anatomy of Annona species. Ann. Bot.84: 771-780.

Pezeshki, S. R. 2001. Wetland plant responses to soil flooding. Environ. Exp. Bot.46: 299- 312.

Vasellati, V., Oesterheld, M., Medan, D. and 
Loreti, J. 2001. Effects of flooding and drought on the anatomy of Paspalum dilatatum. Ann. Bot.88: 355 - 360.

Vincent, E., Robert, D. and Adam, A. 2010.Flooding tolerance of tomato genotypes during vegetative and reproductive stages.Braz. J. Plant Physiol. 22(1): 131-142.

Voesenek, L.A.C.J., Colmer, T.D., Pierik, R., Millenaar, F.F. and Peeters, J.M. 2006. How plants cope with complete submergence. New Phytol. 170: 213226.

Walter, S., Heuberger, H. and Schnitzler, W.S. 2004. Sensibility of different vegetables of oxygen deficiency and aeration with $\mathrm{H}_{2} \mathrm{O}_{2}$ in the rhizosphere. Acta Horticulturae. 659:499-508.

Walter, S., Heuberger, H., and Schnitzler, W. S. 2004. Sensibility of different vegetables of oxygen deficiency and aeration with $\mathrm{H}_{2} \mathrm{O}_{2}$ in the rhizosphere. Acta Horticulturae. 659:499-508.

Wang, S.G., He, L.R., Li, Z.W., Zeng, J.G.,
Chai, Y.R. and Hou, L. 1996. A comparative study on the resistance of barley and wheat to waterlogging. Acta Agron. Sinica. 22:228-232.

Wang, S.G., He, L.R., Li, Z.W., Zeng, J.G., Chai, Y.R. and Hou, L. 1996. A comparative study on the resistance of barley and wheat to waterlogging. Acta Agron. Sinica. 22:228-232.

Yan, B., Dai, Y., Liu, X., Huang, S.andZixia, W. 2006. Flooding induced membrane damage, lipid oxidation and activated oxygen generation in corn leaves.Plant and soil.179(2): 261-268.

Zheng, Y. X., Wu, J. C., Cao, F. L. and Zhang, Y. P. 2010. Effects of water stress on photosynthetic activity, dry mass partitioning and some associated metabolic changes in four provenances of neem (Azadirachta indica A. Juss). Photosynthetica.48:361- 369.

\section{How to cite this article:}

Mohanty. A., R. K. Panda, G. R. Rout, K. C. Muduli and Tripathy. P. 2020. Effect of Short Term Waterlogging on Plant Morphology, Chlorophyll and Carotenoid Content of Tomato (Solanum lycopersicum L. Mill) during Vegetative Stage. Int.J.Curr.Microbiol.App.Sci. 9(07): 920-935. doi: https://doi.org/10.20546/ijcmas.2020.907.107 